\title{
Vagotomy-Induced Enhancement of Mechanical Hyperalgesia in the Rat Is Sympathoadrenal-Mediated
}

\author{
Sachia G. Khasar, ${ }^{1}$ Frederick J.-P. Miao, ${ }^{1}$ Wilfrid Jänig, ${ }^{2}$ and Jon D. Levine ${ }^{1}$ \\ ${ }^{1}$ Departments of Anatomy, Medicine, and Oral and Maxillofacial Surgery, Division of Neuroscience and Biomedical \\ Sciences Program, University of California at San Francisco, San Francisco, California 94143-0452, and 2Physiologisches \\ Institut, Christian-Albrechts-Universität zu Kiel, 24098 Kiel, Germany
}

We have recently shown that subdiaphragmatic vagotomy enhances bradykinin-induced hyperalgesic behavior and decreases baseline paw withdrawal threshold to mechanical stimulation of the hindpaw skin in rats by a peripheral mechanism. To elucidate the underlying mechanism, we studied whether lesions of efferent neuroendocrine pathways could prevent or reverse the potentiating effect of vagotomy. In groups of shamvagotomized or vagotomized rats, we surgically removed or denervated the adrenal medulla. Bradykinin was injected intradermally into the skin of the dorsal surface of the rat hindpaw. Threshold of paw withdrawal to mechanical stimulation of the skin was measured.

Vagotomy induced a decrease in mechanical baseline paw withdrawal threshold and enhancement of bradykinin-induced mechanical hyperalgesic behavior, both of which were maintained over the 5 week testing period. Adrenal enucleation or

In the rat, activity in vagal afferents from abdominal organs modulates transmission of nociceptive impulses in the spinal cord and probably elsewhere in the CNS and also affects experimental pain behavior (Gebhart and Randich, 1992; Randich and Gebhart, 1992; Watkins and Maier, 1995a,b). Recently, we have shown that bradykinin (BK)-induced mechanical cutaneous hyperalgesia is significantly enhanced after subdiaphragmatic vagotomy. A similar effect was seen after cutting the celiac branches of the abdominal vagus nerve but not after cutting only its gastric and hepatic branches. Furthermore, baseline mechanical paw withdrawal threshold decreased after vagotomy. The enhanced mechanical hyperalgesia produced by subdiaphragmatic vagotomy was specific for hyperalgesia induced by BK. Hyperalgesia induced by the direct-acting hyperalgesic agent prostaglandin $\mathrm{E}_{2}$ $\left(\mathrm{PGE}_{2}\right)$ was not affected by vagotomy (Khasar et al., 1997). These findings argue that enhancement of BK-induced mechanical hyperalgesia and decrease in baseline mechanical paw withdrawal threshold after abdominal vagotomy are produced by a peripheral mechanism. However, as the experimental results reported in this manuscript show, we do not exclude a central component in enhancement of BK-induced hyperalgesia after vagotomy, as predicted by the work of Gebhart and Randich (1992) and Randich and Gebhart (1992).

\footnotetext{
Received Oct. 22, 1997; revised Jan. 8, 1998; accepted Jan. 28, 1998.

This work was supported by National Institutes of Health Grant NS 21445.

Correspondence should be address to Dr. Jon D. Levine, Department of Medicine, Box 0452, S-1334, University of California at San Francisco, San Francisco, CA 94143-0452.

Copyright (ㄷ) 1998 Society for Neuroscience $\quad 0270-6474 / 98 / 183043-07 \$ 05.00 / 0$
}

denervation of the adrenal gland by suprarenal ganglionectomy prevented vagotomy-induced decrease in baseline paw withdrawal threshold and enhancement of bradykinin-induced hyperalgesia. In animals that had a demonstrated decrease in baseline paw withdrawal threshold and enhancement of bradykinin-induced hyperalgesia 2 weeks after vagotomy, additional denervation of the adrenal medulla significantly reversed these effects over a 3 week period.

These results imply that both the decrease in baseline paw withdrawal threshold and enhancement of bradykinin-induced hyperalgesic behavior after vagotomy are dependent on a hormonal signal released from the adrenal medulla and suggest a novel mechanism of sensitization of cutaneous nociceptors.

Key words: bradykinin; hyperalgesia; nociception; pain; sympathoadrenal system; vagus

In this study, we tested the hypothesis that vagotomy-induced enhancement of BK-induced mechanical hyperalgesia and decrease in baseline paw withdrawal threshold to mechanical stimulation are mediated via a neuroendocrine mechanism. Our results suggest that the sympathoadrenal system is an essential part of the circuit that mediates these vagotomy-induced hyperalgesic behaviors.

\section{MATERIALS AND METHODS}

The experiments were performed on 46 lightly restrained male Sprague Dawley rats (250-350 gm) purchased from Bantin and Kingman (Fremont, CA) and housed in the animal care facility of the University of California at San Francisco under a $12 \mathrm{hr}$ light/dark cycle. Animal care and use conformed to National Institutes of Health guidelines. Experimental protocols were approved by the University of California at San Francisco Committee on Animal Research. The nociceptive flexion reflex was quantified using a Basile Algesimeter (Stoelting, Chicago, IL) that applies a linearly increasing mechanical force to the dorsum of the rat's hindpaw.

Before using the rats for experiments, they were trained in the paw withdrawal reflex test at 5 min intervals for $1 \mathrm{hr}$ each day for $5 \mathrm{~d}$. This training procedure reduces variability and produces a stable baseline paw withdrawal threshold measurement, thereby enhancing the ability to detect the effect of interventions that modulate nociception (Taiwo et al., 1989). On the day of the experiments, paw withdrawal thresholds (in grams) were measured (i.e., rats were again exposed to the test stimulus) at $5 \mathrm{~min}$ intervals for $1 \mathrm{hr}$. The mean of the last six paw withdrawal thresholds was determined. This mean is defined as the baseline paw withdrawal threshold before the injection of a test agent. BK (0.1-1000 ng) was injected intradermally into the dorsum of both hindpaws in a volume of $2.5 \mu$ l. Thresholds for paw withdrawal were then redetermined at 10,15 , and $20 \mathrm{~min}$ after injection. The mean of the paw withdrawal thresholds obtained at these three time points is the mechanical nociceptive threshold at the dose of BK injected. Increasing doses of BK 
(each greater than the previous dose) were injected cumulatively at 25 -min intervals. Because we have found that injection of small volumes of BK locally in one paw does not affect the threshold of the contralateral paw (our unpublished observation), each paw was treated as an independent measure.

Surgical procedures. Surgery was performed on rats in groups of six. Surgical procedures were performed on half of the group, whereas the other half underwent sham surgery as controls.

Subdiaphragmatic vagotomy. After lateral incision of the abdominal wall in the left upper quadrant, the esophagus was fully exposed at the subdiaphragmatic level (Prechtl and Powley, 1985, 1986; Miao et al., 1994). The vagus nerve was then dissected free from the esophagus and cut bilaterally. A $2.0-2.5 \mathrm{~cm}$ section of the subdiaphragmatic vagus nerve, together with its fine branches, was removed during the surgery. Sham surgery was performed in the same way but without cutting the abdominal vagus nerve. Changes of paw withdrawal thresholds to mechanical stimulation after intradermal BK injections were determined in these rats 7-35 d after vagotomy or sham vagotomy.

Rats were between 250 and $270 \mathrm{gm}$ in weight at the time of subdiaphragmatic surgery. Most vagotomized rats did not display any overt changes in general behavioral pattern. After losing some weight in the first week after the surgery, most of the rats fully recovered. If vagotomized rats did not begin to eat within $24 \mathrm{hr}$ after surgery, then they were unlikely to fully recover; this happened in $\sim 10 \%$ of vagotomized rats. Rats that failed to fully recover from the surgery were excluded from the experiments.

Adrenal medullectomy. To remove adrenal medullas, the adrenal glands were located in ether-anesthetized rats through an incision in the lateral abdominal wall. The capsule of the gland was cut open, and the adrenal medulla was removed (Wilkinson et al., 1981; Miao et al., 1992). Rats were given $0.5 \%$ saline to drink in place of water for the first $7 \mathrm{~d}$ after surgery. Four weeks later, some of the adrenal-medullectomized rats were vagotomized, as described above. Adrenal medullectomy was performed at least 5 weeks before mechanical nociceptive testing. The long postsurgical period was used to allow the function of the hypothalamopituitary-adrenal (HPA) axis to recover; however, in these rats, stressinduced changes in plasma corticosterone levels were still somewhat lower than in normal rats (Wilkinson et al., 1981).

Denervation of the adrenal glands. The greater splanchnic nerve, the adrenal nerves innervating the adrenal gland, the suprarenal ganglion, and the connection to the celiac ganglion were exposed using a retroperitoneal approach after making a lateral incision in the abdominal wall (Celler and Schramm, 1981; Araki et al., 1984). All connections of the suprarenal ganglion were cut, and the ganglion was removed. The wounds were closed. In one group of rats, denervation of the adrenal medullas preceded vagotomy by $7 \mathrm{~d}$. In another group of rats, the two surgeries were done in the same session. In some rats the adrenal glands were denervated 2 weeks after subdiaphragmatic vagotomy. Recovery of the rats was uneventful. Mechanical nociceptive testing in all groups of rats was started $7 \mathrm{~d}$ after surgery.

Groups of rats tested. The groups of rats tested for the different experimental interventions were independent. For each group of rats undergoing vagotomy, removal of the adrenal medulla, or denervation of the adrenal glands, a sham control group was also studied. Rats in which the time course of decrease of paw withdrawal threshold and of enhanced BK-induced mechanical hyperalgesia after vagotomy and their reversal after denervation of the adrenal glands were studied consisted of three subgroups: (1) rats that were only vagotomized and that were repeatedly tested over 5 weeks; (2) rats that were first vagotomized in which the adrenal glands were then denervated 2 weeks later and that were repeatedly tested for 5 weeks after vagotomy; and (3) rats in which the surgical interventions were the same as in group 2 but that were only tested before vagotomy and after denervation of the adrenal glands. An experimental control group of animals that underwent sham vagotomy was tested repeatedly up to day 35 after surgery to show that baseline paw withdrawal threshold and BK-induced mechanical hyperalgesia did not change significantly during repeated testing (see Figs. 4-6).

Statistical analysis. Data are presented as mean \pm SEM and were analyzed statistically using one-factor or repeated-measures ANOVA. The one-factor ANOVA was used to analyze paw withdrawal threshold in the different groups of rats. Data for the vagotomy and sham vagotomy groups at day 7 were the common reference for the different experimental groups. Fisher's protected least significant difference post hoc test was used to determine pairs of groups in which differences occurred. The

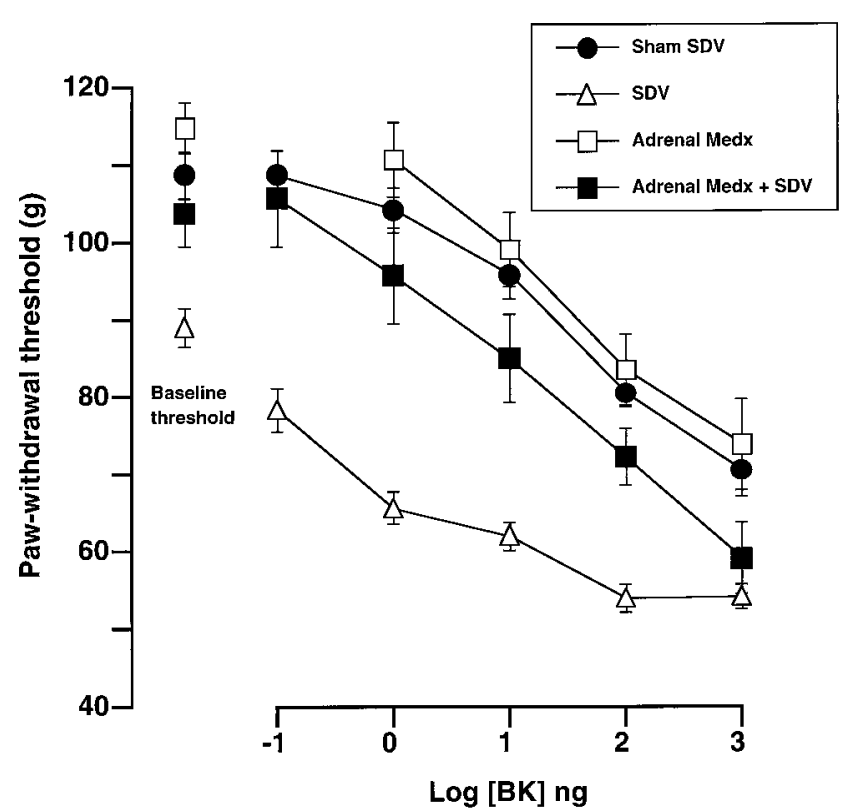

Figure 1. Baseline and decrease of paw withdrawal threshold to mechanical stimulation of the dorsum of the rat hindpaw induced by bradykinin (BK-induced behavioral mechanical hyperalgesia) in sham-vagotomized rats (closed circles; $n=18$ ), in vagotomized rats (open triangles; $n=16$ ), in rats in which adrenal medullas were removed (adrenal medullectomy) (open squares; $n=12$ ), and in rats with removed adrenal medullas and that were also vagotomized (closed squares; $n=12$ ). Experiments were conducted 5 weeks after removal of the adrenal medullas and $7 \mathrm{~d}$ after additional vagotomy. Paw withdrawal thresholds of rats that were only vagotomized and the other three groups of rats were significantly different $(p<0.05)$. Paw withdrawal thresholds of adrenal medullectomized rats and rats that were adrenal medullectomized and also vagotomized were significantly different $(p<0.05)$. Threshold (ordinate scale, in grams) is defined as the force at which the rat withdraws its paw. The abscissa scale is the log dose of BK in nanograms, injected in a volume of $2.5 \mu \mathrm{l}$, into the skin of the dorsal aspect of the hindpaw. In this and subsequent figures: $S D V$, subdiaphragmatic vagotomy; $A M$, adrenal medullas. Shamvagotomized and vagotomized data are the same as those of Khasar et al. (1997), their Figure 1.

repeated-measures ANOVA was used to analyze stimulus-response relationships. The accepted level of significance was $p<0.05$.

\section{RESULTS}

\section{Effect of vagotomy on baseline paw withdrawal threshold and BK-induced hyperalgesia}

Baseline paw withdrawal threshold of sham-vagotomized rats was $108 \mathrm{gm}$ and was significantly decreased to $90 \mathrm{gm} 7 \mathrm{~d}$ after subdiaphragmatic vagotomy (SDV) $(p<0.01$; Fig. 1$)$. Seven days after SDV, intradermal injection of BK produced significantly greater mechanical hyperalgesia compared with its effect in shamvagotomized rats (Fig. 1, compare open triangles, closed circles).

\section{Effect of adrenal medullectomy on baseline paw withdrawal threshold and BK-induced mechanical hyperalgesia in the presence and absence vagotomy}

In adrenal medullectomized rats, vagotomy produced a small but significant reduction in the baseline paw withdrawal threshold and a significant increase in BK-induced hyperalgesia when compared with adrenal medullectomy alone ( $p<0.05$; Fig. 1 , compare closed squares, open squares). Adrenal medullectomized rats that were also vagotomized did not differ significantly from shamvagotomized rats in their baseline paw withdrawal threshold or in response to $\mathrm{BK}$ ( $p>0.05$; Fig. 1 , compare closed squares, closed 
Table 1. Baseline paw withdrawal threshold and paw withdrawal threshold in response to $1 \mathrm{ng}$ of BK injected intradermally into the hindpaw in different groups of rats

\begin{tabular}{|c|c|c|c|c|}
\hline \multirow[b]{2}{*}{ Intervention } & \multirow[b]{2}{*}{$n$} & \multicolumn{2}{|c|}{ Paw Threshold (gm) } & \multirow[b]{2}{*}{ Ref. } \\
\hline & & Baseline & BK (1 ng) & \\
\hline Sham SDV $7 \mathrm{~d}$ & 18 & $107.4 \pm 2.8$ & $104.3 \pm 3$ & in Fig. $1 \bullet$ \\
\hline SDV $7 \mathrm{~d}$ & 20 & $90.7 \pm 1.7^{*}$ & $65.6 \pm 2.5^{*}$ & in Fig. $1 \triangle$ \\
\hline Adrenal medullectomy & 12 & $114.9 \pm 3$ & $110.8 \pm 2.9$ & in Fig. $1 \square$ \\
\hline Adrenal medullectomy + SDV & 12 & $104.0 \pm 2.4^{*}$ & $95.8 \pm 2.1^{*}$ & in Fig. $1 \square$ \\
\hline Adrenal denervation & 6 & $109.2 \pm 3.2$ & $117.2 \pm 4.7$ & in Fig. $2 \square$ \\
\hline Adrenal denervation + SDV & 10 & $105.5 \pm 4.3$ & $100 \pm 6.2^{*}$ & in Fig. $2 \square$ \\
\hline SDV $21 \mathrm{~d}$ & 6 & $86.0 \pm 2.4$ & $61.7 \pm 2.6$ & in Figs. 4, $6 \triangle$ \\
\hline SDV $14 \mathrm{~d}+$ Adrenal denervation $7 \mathrm{~d}$ & 6 & $88.9 \pm 2.3$ & $81.1 \pm 2.3^{*}$ & in Figs. 4, $6 \nabla$ \\
\hline SDV $35 \mathrm{~d}$ & 6 & $81.6 \pm 1.9$ & $57.3 \pm 2.6$ & in Figs. 4, $6 \triangle$ \\
\hline SDV $14 \mathrm{~d}+$ Adrenal denervation $21 \mathrm{~d}$ & 6 & $97.5 \pm 2.8^{*}$ & $94.2 \pm 5.1 *$ & in Figs. 4, $6 \nabla$ \\
\hline
\end{tabular}

SDV, Subdiaphragmatic vagotomy; $n$, Number of observations.

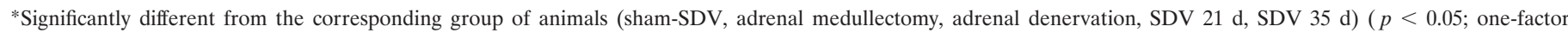
ANOVA).

circles). However, the decrease in paw withdrawal threshold after vagotomy was significantly larger in animals with intact adrenal medullas than in animals with removed adrenal medullas $(p<$ 0.05; Fig. 1, compare closed squares, open triangles). In rats in which adrenal medullas were surgically excised 5 weeks before experiments, baseline paw withdrawal threshold and BK-induced mechanical hyperalgesia were not significantly different compared with sham-vagotomized control rats ( $p>0.05$; Fig. 1, compare open squares, closed circles; Table 1).

\section{Effect of denervation of the adrenal glands on baseline paw withdrawal threshold and BK-induced mechanical hyperalgesia with or without vagotomy}

If the vagotomy-induced decrease in baseline paw withdrawal threshold and increase in BK-induced hyperalgesia are mainly adrenal medulla-mediated phenomena, then denervation of the adrenal glands should mimic the effect produced by adrenal medullectomy; this was indeed the case (compare Figs. 1, 2). When adrenal-denervated rats were vagotomized, BK-induced hyperalgesia was significantly greater when compared with BKinduced hyperalgesia in rats that were only denervated $(p<0.05$; Fig. 2, compare closed squares, open squares). However, this increase in hyperalgesia was much smaller than BK-induced hyperalgesia after vagotomy alone (Fig. 2, compare closed squares, open triangles). In control experiments on rats in which adrenal glands were denervated $7 \mathrm{~d}$ before experiments, the baseline paw withdrawal threshold and BK-induced mechanical hyperalgesia (Fig. 2 , open squares) were not significantly different from those in sham-vagotomized control rats $(p>0.05$; Fig. 2, compare open squares, closed circles; Table 1).

\section{Effect of denervation of the adrenal glands $14 \mathrm{~d}$ after vagotomy}

Although onset of the decrease of baseline mechanical paw withdrawal threshold and enhanced decrease of paw withdrawal threshold to mechanical stimulation generated by intradermal injection of BK was observed as early as $24 \mathrm{hr}$ after vagotomy, these changes progressed slowly and reached peak effects 2-3 weeks after vagotomy. If the decrease of baseline mechanical paw withdrawal threshold and enhanced decrease of paw withdrawal threshold to mechanical stimulation generated by intradermal injection of BK are related to a signal released from the adrenal medullas that is dependent on activity in sympathetic pregangli-

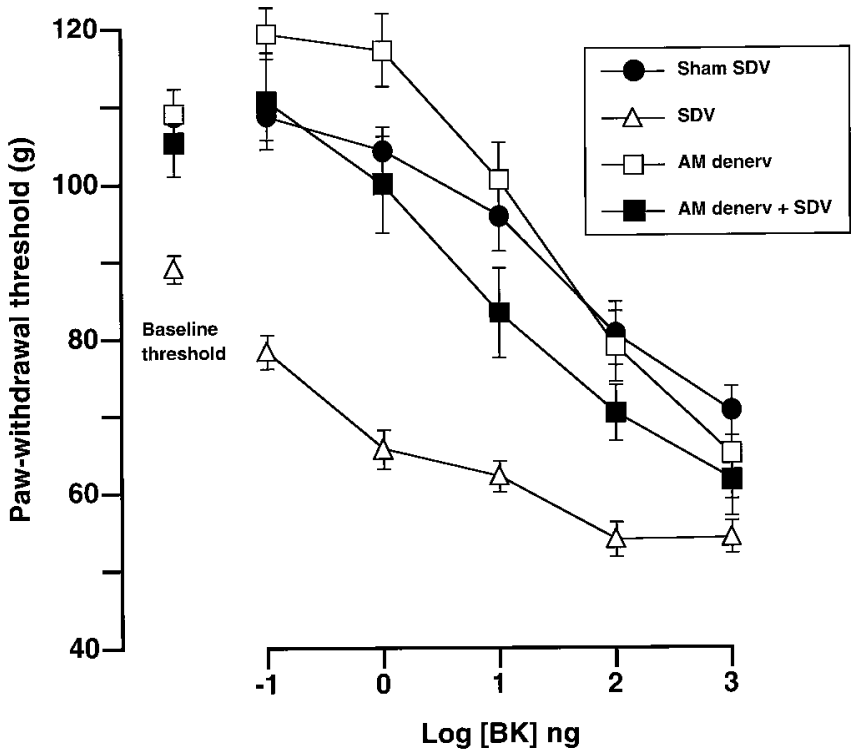

Figure 2. Baseline and decrease of paw withdrawal threshold to mechanical stimulation of the dorsum of the rat hindpaw induced by BK in sham-vagotomized rats (closed circles), vagotomized rats (open triangles), rats with denervated adrenal medullas (open squares; $n=6$ ), and vagotomized rats with denervated adrenal medullas (closed squares; $n=10$ ). In one case, denervation of the adrenal medullas preceded vagotomy by $7 \mathrm{~d}$. In another, vagotomy and denervation of the adrenal medullas were performed in the same session. There was no significant difference in response to BK in these two groups. Therefore, the results were pooled together. Experiments were conducted $7 \mathrm{~d}$ after surgery. Change in paw withdrawal thresholds between animals that were only vagotomized and the other three groups of animals was significantly different $(p<0.05)$. Change in paw withdrawal thresholds between rats with denervated adrenal medullas and vagotomized rats with denervated adrenal medullas was significantly different $(p<0.05)$. Data in sham-vagotomized and vagotomized rats are the same as in Figure 1.

onic axons, then one would expect both changes to be reversed when the adrenal medullas are denervated.

Figure 3 shows that $21 \mathrm{~d}$ after vagotomy ( $7 \mathrm{~d}$ after the denervation of adrenal medullas) there was a significant reversal of vagotomy-induced enhancement of BK mechanical hyperalgesia ( $p<0.05 ;$ compare open inverted triangles, closed squares). The response to $\mathrm{BK}$ on days 28 (data not shown) and 35 after vagot- 


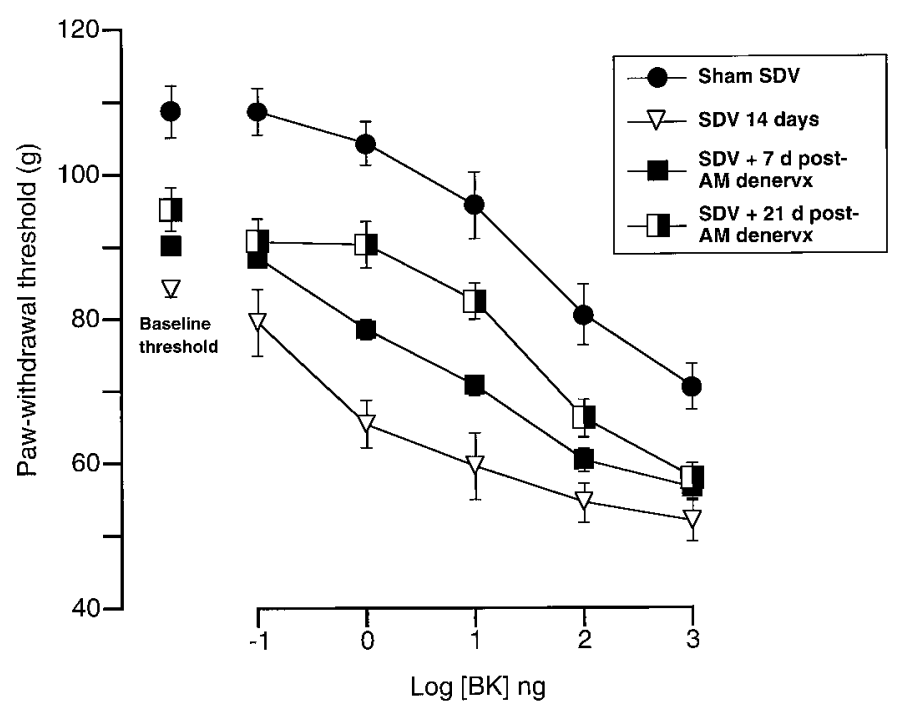

Figure 3. Baseline and decrease of paw withdrawal threshold to mechanical stimulation of the dorsum of the rat hindpaw induced by BK in sham-vagotomized rats (closed circles), vagotomized rats $14 \mathrm{~d}$ after surgery (open inverted triangles; $n=6$ ), and vagotomized rats in which adrenal medullas were also denervated $14 \mathrm{~d}$ after vagotomy $(n=6)$. Experiments were conducted $7 \mathrm{~d}$ (closed squares) or $21 \mathrm{~d}$ (half-closed squares) after denervation of the adrenal medulla in vagotomized animals. The difference between vagotomized rats at $14 \mathrm{~d}$ and rats in which the adrenal medullas were additionally denervated is statistically significant $(p<0.05)$ at 7 and $21 \mathrm{~d}$ after AM denervation.

omy (14 and $21 \mathrm{~d}$ after adrenal medulla denervation) were not significantly different from each other but still significantly different from sham-vagotomized rats $(p<0.05$; Fig. 3, compare half-closed squares, closed circles).

Baseline mechanical paw withdrawal thresholds of vagotomized rats and vagotomized rats in which adrenal medullas were denervated $14 \mathrm{~d}$ after vagotomy are shown in Figure 4. The change in paw withdrawal threshold (i.e., the difference between paw withdrawal threshold elicited by BK and baseline paw withdrawal threshold; Fig. 5, open triangles) and the absolute decrease of paw withdrawal threshold to mechanical stimulation (Fig. 6, open triangles) in response to $1 \mathrm{ng}$ of $\mathrm{BK}$ had a time course similar to the time course of the decrease in baseline paw withdrawal threshold (Fig. 4, open triangles) over a 5 week period. Repeated testing of sham-vagotomized control rats over the same period did not reveal a decrease in paw withdrawal threshold produced by $1 \mathrm{ng}$ of BK (Figs. 5, 6, closed circles). There was reversal of both the decrease in baseline paw withdrawal threshold (Fig. 4) and paw withdrawal threshold attributable to injection of $1 \mathrm{ng}$ of BK (Figs. 5, 6) in vagotomized rats when the adrenal glands were denervated $14 \mathrm{~d}$ after vagotomy. The reversal of both parameters was independent of whether the animals were repeatedly tested in the period between vagotomy and denervation of the adrenal gland or not (Figs. 4-6, half-filled squares, inverted closed triangles). The paw withdrawal thresholds 14 and $21 \mathrm{~d}$ after denervation of the adrenal gland were significantly higher than those measured in the animals that were only vagotomized $(p<0.01$; Figs. 4-6, compare closed triangles, open triangles, Table 1). Furthermore, the differences in paw withdrawal thresholds in response to $1 \mathrm{ng}$ of $\mathrm{BK}$ at 14 and $21 \mathrm{~d}$ after denervation of the adrenal gland in vagotomized animals were not significantly different from those in sham-vagotomized animals that had repeatedly been tested for 5 weeks after surgery (Fig. 5, compare closed triangles, half-closed squares with closed circles).

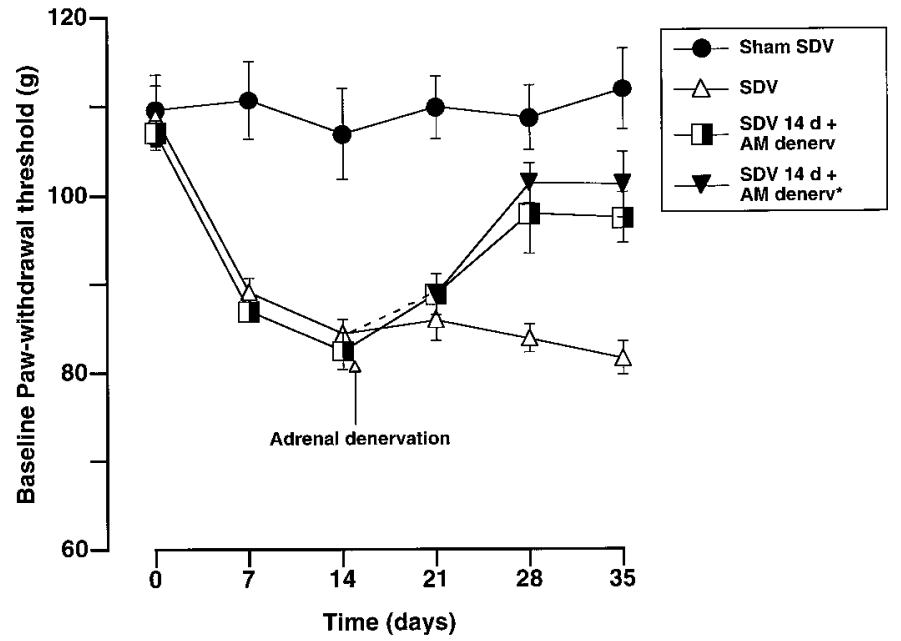

Figure 4. Baseline paw withdrawal threshold in rats before and 7-35 d after vagotomy (open triangles; $n=6$ ) and sham vagotomy (closed circles; $n=8$ ) and in rats that were first vagotomized, followed by denervation of AM $14 \mathrm{~d}$ after vagotomy and measurements taken up to $35 \mathrm{~d}$ after initial surgery. The latter group of animals consists of two subgroups: rats that were tested after vagotomy and after additional denervation of the AM (half-closed squares; $n=6$ ), and rats that were only tested after additional denervation of the adrenal medullas (closed inverted triangles; $n=4$ ). Ordinate scale is threshold in grams. Data of the sham vagotomy and vagotomy group of rats were significantly different $7 \mathrm{~d}$ after vagotomy $(p<0.01)$. Data of vagotomized rats with denervated AM and rats that were only vagotomized were significantly different on days 28 and 35 ( $p<$ 0.01 ). Data between sham-vagotomized rats and vagotomized rats in which the adrenal medullas were denervated were not significantly different on days 28 and $35(p>0.05)$. Also see Figures 5 and 6.

\section{DISCUSSION}

We have recently found that subdiaphragmatic vagotomy in rats is followed by a decrease of baseline mechanical paw withdrawal threshold and by enhancement of mechanical hyperalgesia induced by intradermal injection of BK. This decrease of BKinduced mechanical hyperalgesia involves afferents in the celiac branches of the abdominal vagus nerves (Khasar et al., 1997). Because the hyperalgesic behavior induced by intradermal injection of $\mathrm{PGE}_{2}$ was not affected by subdiaphragmatic vagotomy, we suggest that the enhancement of BK-induced mechanical hyperalgesia was generated by a peripheral mechanism. In the present study we report that decrease of baseline paw withdrawal threshold and enhancement of BK-induced hyperalgesia by subdiaphragmatic vagotomy are dependent on the adrenal medullas and their preganglionic sympathetic innervation. The vagotomyinduced changes do not occur when the adrenal medullas are removed 4 weeks before vagotomy or denervated $7 \mathrm{~d}$ before vagotomy or at the same time as vagotomy is performed. The changes are largely reversed when denervation of the adrenal gland is performed $14 \mathrm{~d}$ after vagotomy. We suggest that vagotomy triggers the activation of sympathetic preganglionic neurons innervating the adrenal medullas (Fig. 7) probably by removing central inhibition acting at this sympathetic pathway, thus leading to the release of a hormonal signal from the adrenal medullas. Interruption of these sympathetic preganglionic axons (by denervation of the adrenal glands) (Fig. 7) stops the release of this hormonal signal and therefore prevents or reverses the decrease of baseline mechanical paw withdrawal threshold and the enhancement of BK-induced hyperalgesia. The hormonal signal released from the adrenal medullas has not yet been identified. 


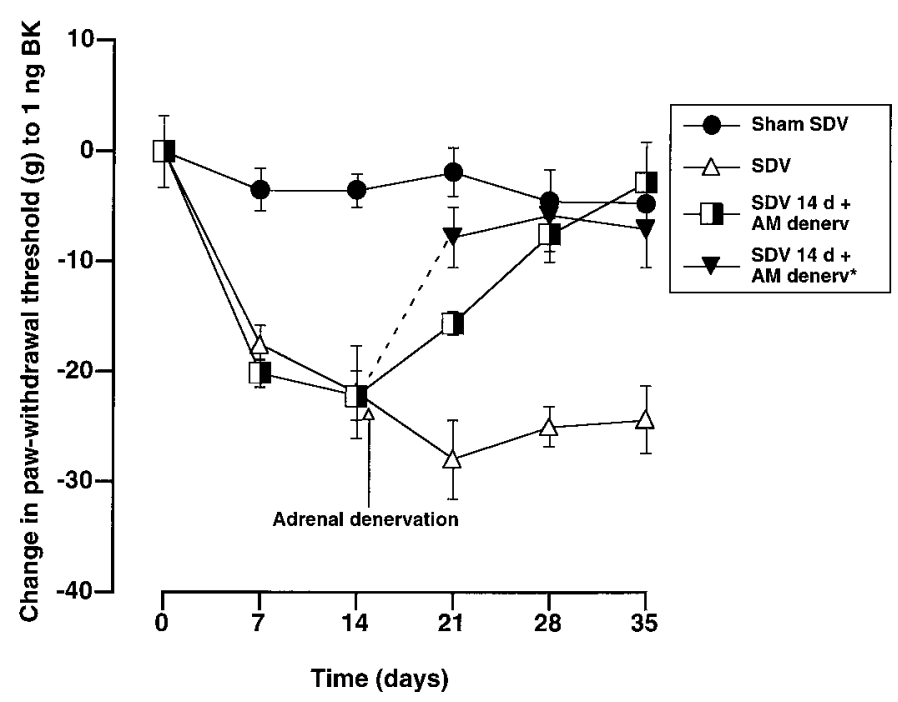

Figure 5. Magnitude of the hyperalgesia (i.e., the decrease in paw withdrawal threshold) induced by $1 \mathrm{ng}$ of BK injected intradermally minus the baseline paw withdrawal threshold for that day (ordinate scale in grams) in rats before and 7-35 days after vagotomy (open triangles), in rats before and 7-35 d after sham vagotomy (closed circles), and in rats that were vagotomized first and in which the AMs were denervated $14 \mathrm{~d}$ after vagotomy and measurements taken up to $35 \mathrm{~d}$ after initial surgery. The latter group of animals consisted of two subgroups: rats that were tested after vagotomy and after additional denervation of the AM (half-closed squares), and rats that were only tested after additional denervation of the adrenal medullas (closed inverted triangles). Data of vagotomized rats with denervated AM and rats that were vagotomized only were significantly different on days 21,28 , and $35(p<0.01)$. Data of sham-vagotomized rats and vagotomized rats in which the adrenal medullas were denervated were not significantly different on days 28 and $35(p>0.05)$.

Candidates include epinephrine, an enkephalin, or an enkephalin-containing neuropeptide that is released on impulse activity in preganglionic sympathetic neurons innervating the adrenal medullas (Jarry et al., 1985; Engeland et al., 1986).

The decrease of paw withdrawal threshold (baseline as well as after intradermal injection of BK) after vagotomy takes several days to reach peak effect, and the recovery after additional denervation of the adrenal medullas takes several days. The reason for the slow time course of these changes is not clear at the moment; we are currently studying the mechanism of this slow time course. The basis of the decrease in paw withdrawal threshold to mechanical stimulation of skin may be a decrease in threshold to mechanical stimulation of all or a subpopulation of cutaneous nociceptors, the recruitment of normally silent (very high threshold) cutaneous afferents (Kress et al., 1992; Jänig and Koltzenburg, 1993; Michaelis et al., 1996), or both. This type of change of sensitivity of a population of cutaneous nociceptors generated by a hormonal signal in which the release is regulated by the brain would be a novel mechanism of sensitization of the population of cutaneous nociceptors.

We have recently shown that activity in vagal afferents that project through the celiac branches of the abdominal vagus nerves and most likely innervate small and large intestines can also change the gain of a nociceptive-neuroendocrine feedback circuit in the control of neurogenic inflammation in the knee joint synovium. Interruption of the vagal afferents enhances the gain of the feedback circuit (Miao et al., 1997a,b). This control circuit acts via the HPA axis to release corticosterone from the adrenal cortex; the sympathoadrenal system does not appear to be in-

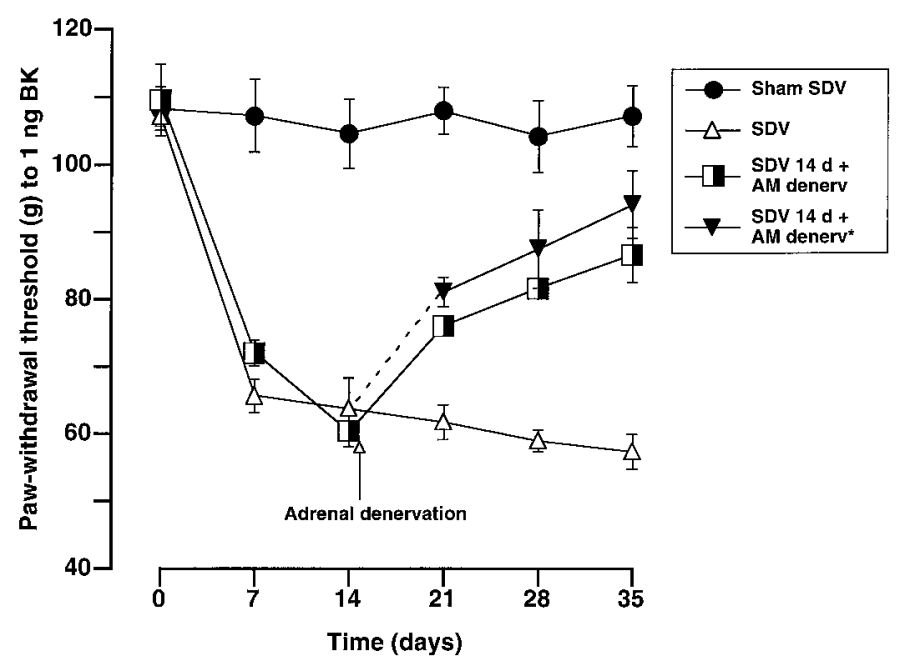

Figure 6. Change of paw withdrawal threshold in response to intradermal injection of $1 \mathrm{ng}$ of bradykinin (1) before and 7-35 d after vagotomy (open triangles), (2) before and 7-14 d after vagotomy and 7-21 d after additional denervation of the adrenal medullas (i.e., 21-35 d after vagotomy; half-closed squares), (3) 21, 28, and 35 d after vagotomy (i.e., 7, 14, and $21 \mathrm{~d}$ after additional denervation of the adrenal medullas; closed inverted triangles), and (4) during repeated testing of a group of shamvagotomized rats over 35 d (closed circles). Data in Figures 4-6 were obtained from the same groups of rats.

volved (Green et al., 1995, 1997). In the current study, vagotomyinduced enhancement of BK mechanical hyperalgesia seems to be under the control of the sympathoadrenal system, and the HPA axis does not appear to be involved (our unpublished data). Thus, activity in vagal abdominal afferents influences two different neural circuits of the neuraxis in the neuroendocrine control of synovial neurogenic inflammation and of cutaneous mechanical hyperalgesia, respectively; one acts through the HPA axis and the central nociceptive system in the neuraxis, and the other acts through the sympathoadrenal axis. A third mechanism by which vagotomy could affect peripheral transduction mechanisms is via the preganglionic input to the postganglionic sympathetic neuron in the lumbar sympathetic chain. However, we have previously shown that interruption of the lumbar sympathetic preganglionic fibers (decentralization of the sympathetic chain) does not affect vagotomy-induced enhancement of BK-induced mechanical hyperalgesia in the rat (Khasar et al., 1997).

Subdiaphragmatic vagotomy is a relatively large intervention that may affect the animal and therefore also nociceptive behavior. That the changes in paw withdrawal threshold and in BKinduced hyperalgesic behavior are nonspecific consequences of vagotomy is unlikely, because (1) the animals tested remained fairly healthy with no overt change in their behavior; (2) cutting the celiac branches of the abdominal vagus nerves also generated enhancement of BK-induced hyperalgesic behavior without a change in baseline threshold; (3) vagotomized animals with removed or denervated adrenal medullas did not exhibit the changes in paw withdrawal threshold to mechanical stimulation; and (4) after denervation of the adrenal medullas in vagotomized animals the changes in paw withdrawal threshold to mechanical stimulation were reversed.

Gebhart and Randich (1992) and Randich and Gebhart (1992) have shown that electrical stimulation of high-threshold abdominal vagal afferents leads to depression of nociceptive impulse transmission of dorsal horn neurons in the lumbar spinal cord and 


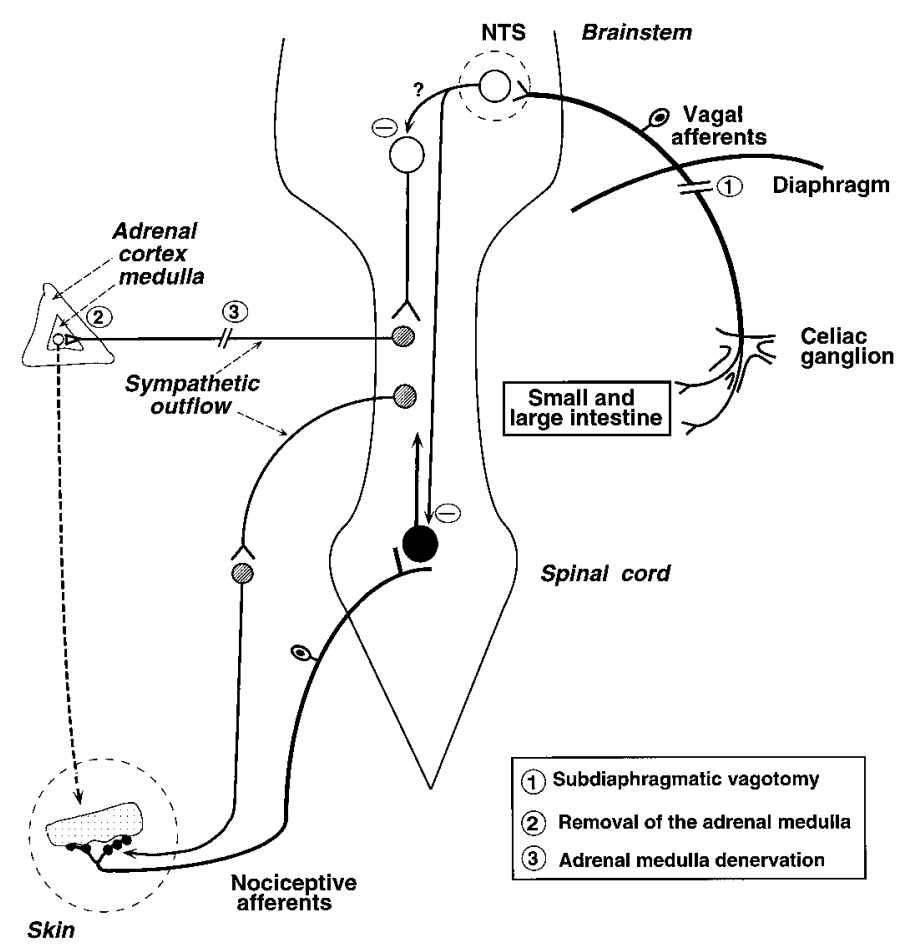

Figure 7. Summary scheme of afferent, central, and efferent pathways that may be involved in decrease of baseline and BK-induced paw withdrawal threshold after subdiaphragmatic vagotomy. Activity in vagal afferents that innervate small and large intestines and project to the nucleus of the solitary tract (NTS) centrally inhibit the pathway to preganglionic neurons innervating the adrenal medullas and neurons of the nociceptive system, e.g., in the dorsal horn. Interruption of these afferents leads to disinhibition of the central pathway to the preganglionic neurons innervating the adrenal medulla and of the central nociceptive system: (1) subdiaphragmatic vagotomy and (2) removal or (3) denervation of the adrenal medullas.

to depression of the tail flick reflex in rats. Similarly, Foreman (1989) has shown that electrical stimulation of cardiopulmonary vagal afferents leads to depression of impulse transmission in spinothalamic neurons that are involved in cardiac nociception. In our behavioral experiments this central effect of vagal activity is normally not seen after vagotomy, at least in part because it is masked by the powerful effect of the endocrine signal released from the adrenal medulla. However, in animals with nonf unctioning adrenal medullas, the mechanical paw withdrawal thresholds to intradermal injection of BK were significantly decreased after vagotomy, although this decrease was smaller than in animals with functioning adrenal medullas (Figs. 1, 2). This suggests that subdiaphragmatic vagotomy has two effects leading to decrease of baseline paw withdrawal threshold and paw withdrawal threshold in response to intradermal injection of $\mathrm{BK}$ : one that is related to the adrenal medulla and its preganglionic sympathetic innervation, and another one that is related to removal of central inhibition of nociceptive impulse transmission occurring probably in the dorsal horn (Fig. 7), as would be predicted from the experiments reported by Gebhart and Randich (1992) and Randich and Gebhart (1992). Thus, we suggest that there are two different central pathways linked to the abdominal vagal afferents: one mediating the influence on nociceptive impulse transmission in the spinal cord, and one influencing activity in sympathetic preganglionic neurons innervating the adrenal medullas (Fig. 7).

As discussed in our previous papers on enhanced BK-induced mechanical hyperalgesia (Khasar et al., 1997) and on neuroendocrine control of BK-induced synovial plasma extravasation (Miao et al., 1997a,b), the changes after vagotomy are generated by the interruption of vagal afferents. This is now corroborated by the observations obtained on animals in which the adrenal medullas were removed or denervated before or after vagotomy. The vagal afferents involved most likely pass through the celiac branches of the abdominal vagus nerves and innervate the small and large intestine (Berthoud and Neuhuber, 1994; Berthoud et al., 1994). Some of them are tonically active (Schwartz and Moran, 1994, 1996). It would be interesting to know whether excitation of these afferents by physiological stimuli increases the mechanical baseline paw withdrawal threshold as well as the mechanical paw withdrawal threshold to intradermal BK.

In conclusion, we have shown that activity in subdiaphragmatic vagal afferents modulates BK-induced mechanical hyperalgesia and baseline mechanical paw withdrawal threshold in the rat. Subdiaphragmatic vagotomy decreases baseline paw withdrawal threshold and paw withdrawal threshold to intradermal injection of BK. Most of this decrease is generated by an endocrine signal released by the adrenal medullas, because denervation or removal of the adrenal medullas prevented these changes. The decrease is large, and paw withdrawal threshold to mechanical stimulation after injection of $1 \mathrm{ng}$ of bradykinin may drop by 40-50 gm (from baseline of $100-110 \mathrm{gm})$. These results may imply that (1) the brain is able to regulate sensitivity of nociceptors all over the body by a neuroendocrine mechanism; (2) sensitivity of nociceptors can be influenced by changes in parts of the body that are remote from the location of the nociceptors; and (3) circulating catecholamines can influence nociceptors in a way that is different from those reported so far (Jänig and McLachlan, 1994; Jänig, 1996; Jänig et al., 1996).

\section{REFERENCES}

Araki T, Ito K, Kurosawa M, Sato A (1984) Responses of adrenal sympathetic nerve activity and catecholamine secretion to cutaneous stimulation in anesthetized rats. Neuroscience 12:289-299.

Berthoud H-R, Neuhuber WL (1994) Distribution and morphology of vagal afferents and efferents supplying the digestive system. In: Innervation of the gut: pathophysiological implications (Taché Y, Wingate DL, Burks TF, eds), pp 43-66. Boca Raton, FL: CRC.

Berthoud H-R, Carlson NR, Powley TL (1991) Topography of efferent vagal innervation of the rat gastrointestinal tract. Am J Physiol 260:200-207.

Celler BG, Schramm LP (1981) Pre- and postganglionic sympathetic activity in splanchnic nerves of rats. Am J Physiol 241:55-61.

Engeland WC, Bereiter DF, Gann DS (1986) Sympathetic control of adrenal secretion of enkephalins after hemorrhage in awake dogs. Am J Physiol 251:341-348.

Foreman RD (1989) Organization of the spinothalamic tract as a relay for cardiopulmonary sympathetic afferent fiber activity. Prog Sens Physiol 9:1-51.

Gebhart GF, Randich A (1992) Vagal modulation of nociception. Am Pain Soc J 1:26-32.

Green PG, Miao FJ-P, Jänig W, Levine JD (1995) Negative feedback neuroendocrine control of the inflammatory response in rats. J Neurosci $15: 4678-4686$.

Green PG, Jänig W, Levine JD (1997) Sympathetic terminal: target for negative feedback neuroendocrine control of inflammatory response in the rat. J Neurosci 17:3234-3238.

Jänig W (1996) The puzzle of "reflex sympathetic dystrophy": mechanisms, hypotheses, open questions. In: Reflex sympathetic dystrophy: a reappraisal (Jänig W, Stanton-Hicks M, eds.), pp 1-24. Seattle: International Association for the Study of Pain.

Jänig W, Koltzenburg M (1993) Pain arising from the urogenital tract. In: The autonomic nervous system, Vol 2, Nervous control of the urogenital system (Maggi CA, ed), pp 523-576. Chur, Switzerland: Harwood Academic. 
Jänig W, McLachlan EM (1994) The role of modifications in noradrenergic peripheral pathways after nerve lesions in the generation of pain. In: Pharmacological approaches to the treatment of pain: new concepts and critical issues. Progress in pain research and management, Vol 1 (Fields HL, Liebeskind JC, eds), pp 101-128. Seattle: International Association for the Study of Pain.

Jänig W, Levine JD, Michaelis M (1996) Interaction of sympathetic and primary afferent neurons following nerve injury and tissue trauma. Prog Brain Res 112:161-184.

Jarry H, Duker EM, Wuttke W (1985) Adrenal release of catecholamines and Met-enkephalin before and after stress as measured by a novel in vivo dialysis method in the rat. Neurosci Lett 60:273-278.

Khasar SG, Miao FJ-P, Jänig W, Levine JD (1997) Modulation of bradykinin-induced mechanical hyperalgesia in the rat by activity in abdominal vagal afferents. Eur J Neurosci, in press.

Kress M, Koltzenburg M, Reeh PW, Handwerker HO (1992) Responsiveness and functional attributes of electrically localized terminals of cutaneous C-fibers in vivo and in vitro. J Neurophysiol 68:581-595.

Miao FJ-P, Helms C, Benowitz NL, Basbaum AI, Heller PH, Levine JD (1992) Chronically administered nicotine attenuates bradykinininduced plasma extravasation and aggravates arthritis-induced joint injury in the rat. Neuroscience 51:649-655.

Miao FJ-P, Jänig W, Dallman MF, Benowitz NL, Heller PH, Basbaum AI, Levine JD (1994) Role of vagal afferents and spinal pathways modulating inhibition of bradykinin-induced plasma extravasation by intrathecal nicotine. J Neurophysiol 72:1199-1207.

Miao FJ-P, Jänig W, Levine JD (1997a) Vagal branches involved in inhibition of bradykinin-induced synovial plasma extravasation produced by intrathecal nicotine and noxious stimulation. J Physiol (Lond) 498:473-481.
Miao FJ-P, Jänig W, Green PG, Levine JD (1997b) Inhibition of bradykinin-induced synovial plasma extravasation produced by noxious cutaneous and visceral stimuli and its modulation by activity in the vagal nerve. J Neurophysiol 78:1285-1292.

Michaelis M, Häbler H-J, Jänig W (1996) Silent afferents: a further class of nociceptors? Clin Exp Pharmacol Physiol 23:14-20.

Prechtl JC, Powley TL (1985) Organization and distribution of the rat subdiaphragmatic vagus and associated paraganglia. J Comp Neurol 235:182-195.

Prechtl JC, Powley TL (1986) Organization and distribution of the rat subdiaphragmatic vagus and associated paraganglia. Brain Res 367:192-200.

Randich A, Gebhart GF (1992) Vagal afferent modulation of nociception. Brain Res Rev 17:77-99.

Schwartz GJ, Moran TH (1994) CCK elicits and modulates vagal afferent activity arising from gastric and duodenal sites. Ann NY Acad Sci 713:121-128.

Schwartz GJ, Moran TH (1996) Sub-diaphragmatic vagal afferent integration of meal-related gastrointestinal signals. Neurosci Biobehav Rev 20:47-56.

Taiwo YO, Levine JD (1989) Prostaglandin effects after elimination of indirect hyperalgesic mechanisms in the skin of the rat. Brain Res 492:397-399.

Watkins LR, Maier SF, Goehler LE (1995a) Cytokine-to-brain communication: a review and analysis of alternative mechanisms. Life Sci 57:1011-1026.

Watkins LR, Maier SF, Goehler LE (1995b) Immune activation: the role of pro-inflammatory cytokines in inflammation, illness responses and pathological pain states. Pain 63:289-302.

Wilkinson CW, Shinsako J, Dallman MF (1981) Return of pituitaryadrenal function after adrenal enucleation or transplantation: diurnal rhythms and responses to ether. Endocrinology 109:162-169. 